is 10 miles I find the r.m.s. error in D.R. position off course would be $\mathrm{I} I \frac{1}{2}$ miles after half an hour, $15 \frac{1}{2}$ miles after one hour and $20 \frac{1}{2}$ miles after $1 \frac{1}{2}$ hours.

\title{
S-Diagrams for Solving Problems in Astronomical Navigation
}

\author{
from Professor W. M. Smart
}

1. THE method described in Schütte's paper ${ }^{1}$ is very ingenious and evidently simple in its application; it depends on solving two pairs of equations of identical forms (in terms of different variables) by simple inspection of diagrams constructed in accordance with the first pair of equations. In his paper the author states, with reference to a figure and without proof, the two pairs of equations referred to. It is obvious that the fourth of Schütte's equations (p. 310 ) is inconsistent with the figure and with the example he gives on the determination of altitude and 'azimuth' (pp. 3 1 2, 313). The trouble arises partly from misconception as to the use of the term azimuth. The method deserves a more detailed explanation of the derivation of the formulae and their application.

2. I shall use the diagram and the notation of Schütte's paper omitting, however, at first any reference to azimuth and replacing $G$ (denoting the heavenly body concerned) by the more familiar $X$. If $H$ is the hour angle, then in the figure $t=360^{\circ}-H$; if the heavenly body is 'west', then $t=H$. It is assumed that $t$, the declination $\delta$ and the latitude $\phi$ are all known; the object is to find the altitude (denoted here by $h$ ) and the true bearing.

From the east point $\mathrm{E}$ (Fig. 1) a great circle is drawn through $\mathrm{X}$ to meet the

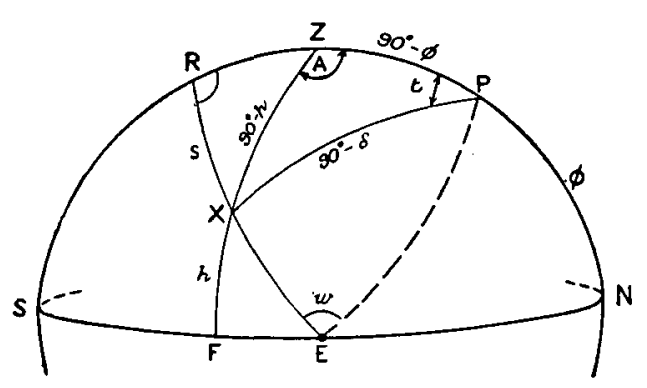

Fig. 1 observer's meridian PZS at R; since $E$ is a pole of $P Z S$, then angle $\mathrm{ZRX}$ is $90^{\circ}$. The arc $\mathrm{XR}$ is $s$ and $o<s<90^{\circ}$ always. In the figure the latitude is north (or positive); the declination may be north or south (that is, positive or negative). There are three principal cases according as $\mathrm{R}$ lies between $S$ and $Z$ (as in the figure), between $Z$ and $P$, and between $P$ and $N$.

The author designates the angle PER-or the arc PR-by $w$.

3. Case $I: R$ between $S$ and $Z$. Here, $o<t<90^{\circ} ; \mathrm{ZR}=\phi+w-90^{\circ} ; \delta$ is + or - . By means of the sine formula and the four-parts formula applied to the triangle PRX we obtain the pair of formulae which give $s$ and $w$ in terms of the known elements $t$ and $\delta$ :

$$
\begin{gathered}
\sin s=\sin t \cos \delta \\
\tan w=\cos t \cot \delta
\end{gathered}
$$

The curves $s=$ constant and $w=$ constant (each drawn on the same chart at intervals of $10^{\prime}$ ) are the curves on which the eventual solutions are based; they are constructed for the ranges $o \leqslant t \leqslant 90^{\circ}$ and $o \leqslant \delta \leqslant 90^{\circ}$. For convenience the 
horizontal scale (the $t$-scale-later called the $x$-scale) is given, in addition, for $t^{\prime}=\mathrm{I} 80^{\circ}-t$ that is, for values between $90^{\circ}$ and $\mathrm{I} 80^{\circ}$.

(i) If $\delta$ is positive, then $s$ and $w$ are, by ( $I$ ) and (2), angles in the first quadrant and, in particular, $\mathrm{PZ}<w<90^{\circ}$ or $w>90^{\circ}-\phi$.

(ii) If $\delta$ is negative, then $\cot \delta$ is negative and hence, by (2), $\tan w$ is negative; then $90^{\circ}<w<\mathrm{PZS}$.

If $\delta_{1}$ denotes the numerical value of the declination and $w_{1}=180^{\circ}-w$, then (2) becomes

also,

$$
\begin{gathered}
\tan w_{1}=\cos t \cot \delta_{1} ; \\
\phi<w_{1}<90^{\circ}
\end{gathered}
$$

For (i) and (ii) $s$ and $w$ or $w_{1}$ are obtained by inspection from the appropriate chart. Schütte writes

then

$$
\begin{aligned}
x_{1}=t, y_{1} & =\delta \text { or } \delta_{1} ; \\
\sin s & =\sin x_{1} \cos y_{1} \\
\tan { }_{w_{1}}^{w} & =\cos x_{1} \cot y_{1}
\end{aligned}
$$

Consider now the triangle $\mathrm{ZRX}$ in which $\mathrm{ZX}=90^{\circ}-h$. Denote the angle PZX by $A$; then angle $R Z X=180^{\circ}-A$. Apply the cosine formula and the fourparts formula; then, since $\mathrm{ZR}=\phi+w-90^{\circ}$, we have

$$
\begin{aligned}
& \sin h=\sin (\phi+w) \cos s \\
& \cot A=\cos (\phi+w) \cot s
\end{aligned}
$$

From the figure, $\phi+w=\mathrm{NP}+\mathrm{PR}=90^{\circ}+\mathrm{ZR}$ so that $\phi+w>90^{\circ}$ and $\cos (\phi+w)$ is negative; consequently $\cot A$ is negative, that is, $90^{\circ}<A<180^{\circ}$. Put

Then (7) becomes

$$
\mathrm{A}=90^{\circ}+a
$$

$$
\tan a=\cos \left\{180^{\circ}-(\phi+w)\right\} \cot s
$$

When $\delta$ is - and $\mathrm{w}_{1}=180^{\circ}-w$, this last equation becomes

$$
\tan a=\cos \left(w_{1}-\phi\right) \cot s
$$

In Schütte's notation put

and

$$
x_{2}=180^{\circ}-(\phi+w) \text {, when } \delta \text { is }+
$$

and

$$
x_{2}=w_{1}-\phi \text {, when } \delta \text { is - }
$$

Since $180^{\circ}>\phi+w$, then $w_{1}>\phi$.

Then (6) and (9) or ( 10 ) become

$$
\begin{aligned}
\sin h & =\sin x_{2} \cos y_{2} \\
\tan a & =\cos x_{2} \cot y_{2}
\end{aligned}
$$

These formulae have the same forms as (4) and (5) and so $h$ and $a$ (both between $0^{\circ}$ and $90^{\circ}$ ) can be obtained from the appropriate chart with the $x$-coordinate corresponding to $(\phi+w)$ when $\delta$ is + or to $\left(w_{1}-\phi\right)$ when $\delta$ is -.

4. Case II: $R$ between $Z$ and P. Here, $o<t<90^{\circ} ; \delta$ is $+; \mathrm{ZR}=90^{\circ}-(\phi+w)$. It is easily seen by the procedure in para. 3 that

where

$$
\begin{aligned}
\sin s & =\sin x_{1} \cos y_{1} \\
\tan w & =\cos x_{1} \cot y_{1}, \\
x_{1} & =t, y_{1}=\delta ;
\end{aligned}
$$


also,

$$
\sin h=\cos Z R \cos s=\sin (\phi+w) \cos s
$$

$$
\cot A=\sin Z R \cot s=\cos (\phi+w) \cot s
$$

where $A=$ angle $P Z X$ and $o<A<90^{\circ}$. Put

and $x_{2}=\phi+w, y_{2}=s$; then

$$
\mathrm{A}=90^{\circ}-a
$$

$$
\begin{aligned}
\sin h & =\sin x_{2} \cos y_{2} \\
\tan a & =\cos x_{2} \cot y_{2}
\end{aligned}
$$

In this case, that is,

$$
\begin{gathered}
\phi+w=N P+P R \text { so that } \phi x_{2}<90^{\circ}, \\
w<90^{\circ}-\phi .
\end{gathered}
$$

5. Case III: $R$ between $P$ and $N$. Here, $90^{\circ}<t<180^{\circ} ; \delta$ is + and ZR $=90^{\circ}$ $-\phi+w$.

In triangle $\mathrm{XPR}$, angle $\mathrm{XPR}=180^{\circ}-t$ and then

where

$$
\begin{aligned}
\sin s & =\sin \left(180^{\circ}-t\right) \cos \delta=\sin x_{1} \cos y_{1} \\
\tan w & =\cos \left(180^{\circ}-t\right) \cot \delta=\cos x_{1} \cot y_{1}
\end{aligned}
$$

So far as deriving the elements $s$ and $w$ from the appropriate chart is concerned we can use $x_{1}=t$ as the $x$-coordinate.

In this case, $o<A<90^{\circ}$. Put

$$
A=90^{\circ}-a
$$

and then we easily find that

where

$$
\begin{aligned}
& \sin h=\sin x_{2} \cos y_{2} \\
& \tan a=\cos x_{2} \cot y_{2}
\end{aligned}
$$

Also,

$$
x_{2}=\phi-w \text { and } y_{2}=s \text {. }
$$

$$
w<\phi \text {. }
$$

6. Summary of rules. In all cases it is assumed that $s, w$ (or $w_{1}$ ), $h$ and $a$ (all are angles between $0^{\circ}$ and $90^{\circ}$ ) have been found from the charts with the appropriate values of $x_{1}, y_{1}, x_{2}$ and $y_{2}$.

I. $\delta-; o<t<90^{\circ}$

Then $w_{1}>\phi$ and $A=90^{\circ}+a$ (Case I (ii))

2. $\delta+; o<t<90^{\circ}$

(I) If $\phi+w>90^{\circ}$, then $A=90^{\circ}+a$ (Case I (i))

(2) If $\phi+w<90^{\circ}$, then $A=90^{\circ}-a$ (Case II)

3. $\delta+; 90^{\circ}<t<180^{\circ}$

$$
\text { Then } w<\phi \text { and } A=90^{\circ}-a \text { (Case III) }
$$

It is to be remarked that the formulae obtained are the foundations of Aquino's and Ageton's tables.

In each case, for north latitude, the true bearing (T.B.) is easily obtained.

For, if the heavenly body is 'east' as in the figure,

$$
\text { T.B. }=\text { A }
$$

and, if the heavenly body is 'west',

$$
\text { T.B. }=360^{\circ}-\mathrm{A}
$$


If the latitude is south, the T.B. can be derived in a similar way; thus, for heavenly body 'east',

$$
\text { T.B. }=180^{\circ}-\mathrm{A}
$$

and, for 'west',

$$
\text { T.B. }=180^{\circ}+A
$$

7. Schütte's Example (p. $3^{1} 2$ ). It is found, with the values of $\phi$ and $w$, the latter obtained from the charts, that $\phi+w<90^{\circ}$; hence, by 2 (II) of para. 6 , the example belongs to Case II-as can also be seen easily from a figure. Then

$$
A=90^{\circ}-a=64^{\circ} 26^{\prime}
$$

so that

$$
\text { T.B. }=064^{\circ} 26^{\prime}
$$

Now, Azimuth is a term necessarily related to the celestial horizon as reference-plane and to some point on it as reference-point. According to Schütte's figure this reference-point is $S$, in which event the true bearing should be $180^{\circ}-64^{\circ} 26^{\prime}$, which is wrong.

If, on the other hand, Schütte defines Azimuth as the angle RZX in the triangle $\mathrm{RZX}$ then his value of Az. is $64^{\circ} 26^{\prime}$ and the true bearing is $064^{\circ} 26^{\prime}$. But this definition depends on a particular construction (involving $R$ ) and it has no general significance in the ordinary problem associated with the fundamental spherical triangle PZX; such a definition inevitably leads to confusion.

The moral seems to be that there should be a consistent definition of the term Azimuth and this is achieved by defining it-as most textbooks do-as the angle $\mathrm{PZX}$ in the spherical triangle PZX; after all, this angle is directly connected with the usual formulae for a spherical triangle, and from it the corresponding true bearing can be very easily deduced.

\section{REFERENCES}

1 Schütte, K. (1955). S-diagrams for solving problems in astronomical navigation. This Journal, 8, 3 I0.

\section{The English and the Dutchman's Log}

$$
\text { from E. G. R. Taylor }
$$

IN his detailed and valuable account of the history of the Log (this Journal, 9, 70) Commander Waters is surely at fault as regards the part he ascribes to the Gresham Professor, Gunter. This eminent mathematician delighted in designing instruments with engraved scales, and at first issued the customary Description and Use in Latin, a sufficient indication that he wrote only for fellow scholars. Importuned probably by the instrument-makers, he later published English versions of the tracts (in 1623), and among the numerous 'uses' included a couple of chapters on those for navigational purposes. Two and a half pages in the Second Book of the Cross-staff (Chapter VI, Section I) are given to 'the ship's way', but there is no suggestion that any novelty of method is being 